\title{
Korean Guidelines for the Pharmacological Treatment of Social Anxiety Disorder: Initial Treatment Strategies
}

\author{
Hyungkun Yoon ${ }^{1}$, Dong Jae Oh², Ho-Suk Suh³, Kyoung-Uk Lee ${ }^{4}$, Se-Won Lim ${ }^{5}$, Jun-Yeob Lee, \\ Jong-Chul Yang ${ }^{7}$, Jae-Hon Lee ${ }^{8,9}$, Juwon $\mathrm{Ha}^{10}$, Bun-Hee Lee ${ }^{11}$, Seung-Gul Kang ${ }^{12}$, Ho-Kyoung Yoon ${ }^{13}$, \\ Jihyun Moon ${ }^{2}$, Seung-Min Bae ${ }^{12}$, Youngdo Kwon ${ }^{14}$, Hyun-Chung Kim ${ }^{15}$, and Kang Seob $\mathrm{Oh}^{5} \bowtie$ \\ ${ }^{1}$ Workplace Mental Health Institute, Kangbuk Samsung Hospital, Sungkyunkwan University School of Medicine, Seoul, Republic of Korea \\ ${ }^{2}$ Miso Psychiatric Clinic, Seoul, Republic of Korea \\ ${ }^{3}$ Department of Psychiatry, School of Medicine, CHA University, CHA Gangnam Medical Center, Seoul, Republic of Korea \\ ${ }^{4}$ Department of Psychiatry, Uijeongbu St. Mary's Hospital, The Catholic University of Korea, Uijeongbu, Republic of Korea \\ ${ }^{5}$ Department of Psychiatry, Kangbuk Samsung Hospital, Sungkyunkwan University School of Medicine, Seoul, Republic of Korea \\ ${ }^{6}$ Saebit Hospital, Gyeongju, Republic of Korea \\ ${ }^{7}$ Department of Psychiatry, Chonbuk National University Hospital, Jeonju, Republic of Korea \\ ${ }^{8}$ Department of Psychiatry, Korea University Ansan Hospital, Korea University College of Medicine, Ansan, Republic of Korea \\ ${ }^{9}$ Mood Disorders Psychopharmacology Unit, University Health Network, Toronto, Ontario, Canada \\ ${ }^{10}$ Yonsei Forest Mental Health Clinic, Seoul, Republic of Korea \\ ${ }^{11}$ Maum \& Maum Clinic, Seoul, Republic of Korea \\ ${ }^{12}$ Department of Psychiatry, Gil Medical Center, Gachon University, School of Medicine, Incheon, Republic of Korea \\ ${ }^{13}$ Department of Psychiatry, Korea University College of Medicine, Seoul, Republic of Korea \\ ${ }^{14}$ Smart Psychiatry Clinic, Sejong, Republic of Korea \\ ${ }^{15}$ Department of Psychiatry, National Medical Center of Korea, Seoul, Republic of Korea
}

Objective The aim of the present study was to provide clinical consensus and evidence regarding initial treatment strategies for the pharmacological treatment of social anxiety disorder (SAD) in Korea.

Methods We prepared a questionnaire to derive a consensus from clinicians regarding their preference for the pharmacological treatment of SAD in Korea. Data regarding medication regimens and psychotropic drugs used during initial treatment, the doses used, and the pharmacological treatment duration were obtained. Responses were obtained from 66 SAD experts, and their opinions were classified into three categories (first-line, second-line, third-line) using a chi-square analysis.

Results Clinicians agreed upon first-line regimens for SAD involving monotherapy with selective serotonin reuptake inhibitors (SSRIs) or the serotonin-norepinephrine reuptake inhibitor (SNRI) venlafaxine, or combined therapy using antidepressants with betablockers or benzodiazepines on a standing or as-needed basis. First-line psychotropic drug choices for initial treatment included the following: escitalopram, paroxetine, sertraline, venlafaxine, and propranolol. The medication dosage used by domestic clinicians was found to be comparable with foreign guidelines. Domestic clinicians tended to make treatment decisions in a shorter amount of time and preferred a similar duration of maintenance treatment for SAD when compared with foreign clinicians.

Conclusion This study may provide significant information for developing SAD pharmacotherapy guidelines in Korea, especially in the early stage of treatment.

Psychiatry Investig 2018;15(2):147-155

Key Words Social anxiety disorder, Guideline, Initial treatment, Pharmacotherapy.

\section{INTRODUCTION}

Social anxiety disorder (SAD) is characterized by dysfunction in daily life due to the extreme fear of social situations, in which affected individuals experience embarrassment, shame, and feelings of powerlessness, in addition to continued avoidance of social situations. ${ }^{1}$ Since shyness and modesty are accepted and even honored in Asian culture, SAD is

Received: January 5, 2017 Revised: March 15, 2017 Accepted: May 1, 2017 Available online: November 29, 2017

$\bowtie$ Correspondence: Kang Seob Oh, MD, PhD

Department of Psychiatry, Kangbuk Samsung Hospital, Sungkyunkwan University School of Medicine, 29 Saemunan-ro, Jongno-gu, Seoul 03181, Republic of Korea Tel: +82-2-2001-2214, Fax: +82-2-2001-2211, E-mail: ks2485@empas.com

(a) This is an Open Access article distributed under the terms of the Creative Commons Attribution Non-Commercial License (http://creativecommons.org/licenses/by-nc/4.0) which permits unrestricted non-commercial use, distribution, and reproduction in any medium, provided the original work is properly cited. 
less likely to be recognized as a pathology in Asian countries. ${ }^{2,3}$ However, considering the high prevalence (7-13\%) of individuals who experience functional disability related to SAD in the Western world, ${ }^{4,5}$ the incidence in Eastern countries is likely to be higher than previously reported. Clinical diagnosis and treatment for SAD is crucial due to the relatively early age of onset and the tendency for symptoms to develop into chronic conditions. Untreated SAD can eventually result in severe social and occupational impairment, often leading to co-morbid psychiatric disorders such as major depressive disorder. ${ }^{6}$

Common treatments for SAD include cognitive behavioral therapy (CBT), pharmacological intervention, or a combination of CBT and medication. While pharmacotherapy has been shown to be effective in improving irrational fear and avoidance behaviors, such treatment is limited in that the choice of medication depends on the subjective experience and knowledge of individual clinicians, as a number of psychotropic drugs have been developed for the treatment of various mental health conditions, including SAD. In order to make more rational decisions regarding the most appropriate treatment for each patient, clinically proven guidelines for practice are necessary. ${ }^{7}$

Such guidelines have been developed and used in a variety of countries, ${ }^{8-13}$ although there exists a lack of research and consensus regarding such guidelines in Korea. Thus, we aimed to develop guidelines for the pharmacological treatment of SAD in Korea with the support of the Korean Academy of Anxiety Disorder, and to provide information regarding initial treatment strategies for the development of such a guideline.

\section{METHODS}

\section{Development of the guideline questionnaire}

Research on the pharmacological treatment of SAD in Korea is scarce. Thus, we developed a questionnaire in order to identify points of agreement among clinical experts. We reviewed both domestic and foreign guidelines and sought the advice of researchers who had developed these guidelines. We reviewed guidelines developed by the World Federation of Societies of Biological Psychiatry (WFSBP), ${ }^{8,14}$ National Institute for Health and Clinical Excellence (NICE), ${ }^{15}$ British Association for Psychopharmacology (BAP), ${ }^{13}$ Canadian Psychiatric Association (CPA), ${ }^{9}$ and European College of Neuropsychopharmacology (ECNP) ${ }^{10}$ among others. The questionnaire was prepared focusing on the WFSBP guideline ${ }^{8}$ that had been most recently developed based on scientific evidence at the time of developing the questionnaire, and the final draft was developed following repeated review and modified to fit the domestic situation based on the results of a preliminary survey.
The questionnaire was configured according to the Diagnostic and Statistical Manual of Mental Disorders, 4th edition, Text Revision (DSM-IV-TR), the diagnostic criteria at the time of data collection. Based on DSM-IV-TR diagnostic criteria, SAD was often categorized into generalized and non-generalized subtypes. However, because it was unnecessary to use both subtypes in creating effective guidelines and distinction between the two subtypes were deleted in the DSM-5 criteria, this study was confined to the generalized specifier.

A list of psychotropic drugs marketed and used to treat SAD in Korea as of March 2013 was included with the questionnaire in order to assess the experiences of each clinician in prescribing these drugs. The duration of pharmacological intervention and the dosage of medication were either chosen from the list or manually entered by respondents. Respondents were instructed to choose the ideal treatment for improving symptoms of SAD and enhancing patient quality of life. Due to economic limitations and institutional situations, psychotropic drugs that are more appropriate and ideal from a medical standpoint may differ from those prescribed in actual clinical situations. We therefore placed emphasis on the selection of "ideal or preferable" treatment options, rather than "realistic" options.

The questionnaire included the following four questions regarding initial treatment strategies for patients with SAD without comorbidity: 1) What regimen was preferred as the initial medication? 2) What type of pharmacological intervention was actually chosen at this stage? 3) What were the starting, maintenance, and maximum doses for the initial pharmacological treatment?, and 4) How long was the evaluation period after the initial pharmacological treatment, and what were the durations of maintenance therapy, the tapering phase, and treatment for $\mathrm{SAD}$ recurrence?

\section{Organization of the review committee and collection of survey responses}

The review committee was formed of experts chosen from among researchers who published papers related to SAD and the pharmacological treatment of anxiety disorders, members of existing major Korean psychiatric pharmacological treatment guideline committees, officers of the Korean Academy of Anxiety Disorder, officers of the Korean College of Neuropsychopharmacology, and other neuropsychiatric practitioners with extensive clinical experience in SAD.

A total of 218 review committee members were asked to fill out the questionnaire, 66 of whom responded. Among these 66 respondents were 42 professors of university hospitals, 15 specialists in general and psychiatric hospitals, and nine practitioners from private hospitals. Members who replied to the questionnaire received a small amount of mone- 
tary compensation. All participants consented to participate in this study.

\section{Analysis of questionnaire results}

In order to evaluate preferred regimens and drugs, respondents were asked to rate the appropriateness of each treatment selection along a 9-point scale (1-9). When lacking enough experience and recognition of a certain item, they were instructed to instead mark the question with a "(q)" (Figures 1 and 2).

First, consensus was determined by investigating differences in the distribution of responses among the three categories of appropriateness (1-3, 4-6, and 7-9) using the chisquare test, following which mean values and $95 \%$ confidence intervals were calculated. Based on the $95 \%$ confidence interval of each sub-item, responses were separated into three categories: first-line/preferred choice, second-line/alternative choice, and third-line/unsuitable choice. First- and secondline selections were divided based on the scores of the lowest boundary of the confidence interval. Scores of 6.5 or higher were indicative of first-line selections, while scores from 3.5 to 6.5 were indicative of second-line selections, and scores lower than 3.5 were indicative of third-line selections. When the confidence interval of a second-line selection overlapped with that of first-line selections, the selection was regarded as upper second-line. When the confidence interval did not overlap, it was regarded as a lower second-line selection. When the distribution of expert opinions did not differ among the three categories of the chi-square test, the result was marked as "no consensus." Such results indicated that the opinion dis- tribution of the review committee was relatively uniform, thus resulting in a lack of clear consensus regarding the most appropriate treatment.

When a survey item received a high number of “( )" responses from 66 survey respondents (when $\mathrm{N}$ was less than 30 ), the item was excluded from reporting.

\section{Review of results and creation of guidelines}

The questionnaire results were used by the authors to revise guidelines for the treatment of SAD in accordance with the current domestic situation in Korea, in which there were not enough number of experts for the clinical treatment of SAD. A public hearing was held to maximize fairness. After the results of the public hearing were further reviewed by three experts, the guidelines were produced and presented.

\section{RESULTS}

\section{Initial treatment regimens}

For the initial treatment of SAD without comorbidity, respondents agreed upon two regimens as first-line options: 1) monotherapy with selective serotonin reuptake inhibitors (SSRIs) and 2) concomitant use of an antidepressant with a beta-blocker or benzodiazepine on either a standing or an asneeded basis in accordance with the clinician's judgement. When triple therapy consisting of antidepressants, beta-blockers, and benzodiazepines was agreed upon, the use of betablockers on an as-needed basis was considered as a first-line option, while the use of beta-blockers on a standing basis was considered as an upper second-line option (Table 1).

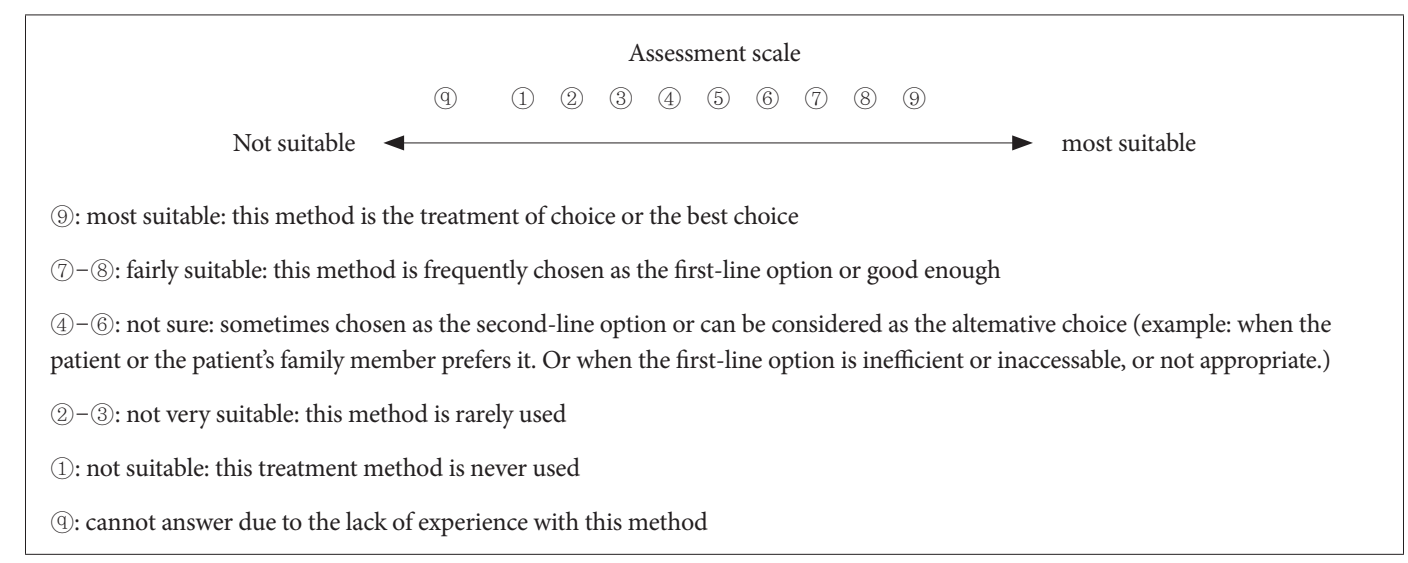

Figure 1. Explanations regarding the level of assessment in the questionnaire.

Assessment item

1. SSRI only monotherapy

2. Benzodiazepine-class anxiolytics monotherapy
Assessment criterion

(1) (1) (2) (3) (4) (5) (6) (7) (8) (9)

(4) (1) (2) (3) (4) (5) (6) (7) (8) (9)

Figure 2. Examples of assessment in the questionnaire. 
SSRI monotherapy was agreed upon as a first-line option, whereas serotonin-norepinephrine reuptake inhibitor (SNRI) monotherapy was agreed upon as an upper second-line option. Many foreign guidelines regard venlafaxine as the firstline pharmaceutical choice, and SNRI was specified as the firstline recommendation in this case and was witten as "SNRI (venlafaxine)." Therefore, a final agreement was made among the authors that the prescription of "SNRI (venlafaxine)" was to be included and recommended as a first-line option as well.

Monotherapy with beta-blockers or benzodiazepines without an antidepressant was agreed upon as a lower second-line choice in all cases, and all antidepressants other than SSRIs and SNRIs were also agreed upon as lower second-line options.

All anticonvulsants were regarded as third-line options or had received a high number of “(q)" responses $(n<30)$.

\section{Psychotropic drugs used in the initial stages of treatment}

Among drugs utilized for initial treatment, escitalopram, paroxetine, sertraline, venlafaxine, duloxetine, and propranolol were agreed upon as first-line choices (Table 2).

In the survey of initial treatment regimens, SNRI antidepres-

Table 1. Preferred regimens for the initial treatment of social anxiety disorder

\begin{tabular}{|c|c|c|c|c|c|c|}
\hline \multirow{2}{*}{ Treatment regimens } & \multirow{2}{*}{ Mean } & \multirow{2}{*}{$\mathrm{SD}$} & \multicolumn{2}{|c|}{$95 \%$ confidence interval } & \multirow{2}{*}{$\begin{array}{l}\text { Score } \\
9(\%)\end{array}$} & \multirow{2}{*}{ p-value } \\
\hline & & & Lower & Upper & & \\
\hline \multicolumn{7}{|l|}{ First-line } \\
\hline SSRI only & 7.6 & 0.2 & 7.2 & 7.9 & 30.3 & 0.000 \\
\hline Antidep $+\beta$-blocker as & 7.3 & 0.2 & 7.0 & 7.6 & 18.2 & 0.000 \\
\hline Antidep+BDZ st & 7.2 & 0.2 & 6.9 & 7.6 & 21.2 & 0.000 \\
\hline Antidep+BDZ as & 7.2 & 0.2 & 6.9 & 7.6 & 21.2 & 0.000 \\
\hline Antidep $+\mathrm{BDZ}$ st $+\beta$-blocker as & 6.9 & 0.2 & 6.6 & 7.3 & 15.2 & 0.000 \\
\hline Antidep $+\mathrm{BDZ}$ as $+\beta$-blocker as & 6.9 & 0.2 & 6.5 & 7.3 & 18.2 & 0.000 \\
\hline Antidep $+\beta$-blocker st & 6.9 & 0.2 & 6.5 & 7.2 & 12.1 & 0.000 \\
\hline \multicolumn{7}{|l|}{ Upper second-line } \\
\hline SNRI only & 6.8 & 0.2 & 6.4 & 7.2 & 13.6 & 0.000 \\
\hline Antidep+BDZ st $+\beta$-blocker st & 6.6 & 0.2 & 6.2 & 7.0 & 9.1 & 0.000 \\
\hline Antidep $+\mathrm{BDZ}$ as $+\beta$-blocker st & 6.5 & 0.2 & 6.0 & 6.9 & 6.1 & 0.000 \\
\hline \multicolumn{7}{|l|}{ Lower second-line } \\
\hline NaSSA only & 5.5 & 0.2 & 5.2 & 5.9 & 0.0 & 0.000 \\
\hline Buspirone & 5.3 & 0.2 & 4.9 & 5.8 & 0.0 & 0.000 \\
\hline$\beta$-blocker as only & 4.9 & 0.3 & 4.4 & 5.4 & 4.6 & 0.156 \\
\hline $\mathrm{BDZ}$ as $+\beta$-blocker as & 4.9 & 0.3 & 4.4 & 5.5 & 4.6 & 0.666 \\
\hline RIMA only & 5.0 & 0.4 & 4.3 & 5.7 & 4.6 & 0.000 \\
\hline $\mathrm{BDZ}$ st $+\beta$-blocker st & 4.8 & 0.2 & 4.3 & 5.3 & 1.5 & 0.001 \\
\hline $\mathrm{BDZ}$ as only & 4.6 & 0.3 & 4.1 & 5.2 & 3.0 & 0.002 \\
\hline $\mathrm{BDZ}$ st $+\beta$-blocker as & 4.6 & 0.2 & 4.1 & 5.0 & 1.5 & 0.000 \\
\hline Atypical antipsychotics & 4.5 & 0.2 & 4.1 & 5.0 & 0.0 & 0.000 \\
\hline $\mathrm{BDZ}$ as $+\beta$-blocker st & 4.5 & 0.2 & 4.1 & 4.9 & 0.0 & 0.000 \\
\hline$\beta$-blocker st only & 4.2 & 0.2 & 3.7 & 4.7 & 0.0 & 0.000 \\
\hline BDZ st only & 4.2 & 0.2 & 3.7 & 4.6 & 0.0 & 0.000 \\
\hline TCA only & 4.1 & 0.2 & 3.6 & 4.5 & 0.0 & 0.000 \\
\hline \multicolumn{7}{|l|}{ Third-line (not suitable) } \\
\hline Anticonvulsant & 3.0 & 0.2 & 2.5 & 3.5 & 0.0 & 0.000 \\
\hline Typical antipsychotics & 1.9 & 0.2 & 1.6 & 2.2 & 0.0 & 0.000 \\
\hline
\end{tabular}

SSRI: selective serotonin reuptake inhibitor, Antidep: antidepressant, $\beta$-blocker as: $\beta$-blocker as-needed basis, BDZ: benzodiazepine, BDZ st: $\mathrm{BDZ}$ standing basis, $\mathrm{BDZ}$ as: $\mathrm{BDZ}$ as-needed basis, $\beta$-blocker st: $\beta$-blocker standing basis, SNRI: serotonin-norepinephrine reuptake inhibitor, NaSSA: noradrenergic and specific serotonergic antidepressant, RIMA: reversible inhibitor of monoamine oxidase A, TCA: tricyclic antidepressant 
$\mathrm{H}$ Yoon et al.

Table 2. Preferred drugs for the initial treatment of social anxiety disorder

\begin{tabular}{|c|c|c|c|c|c|c|c|}
\hline \multirow{2}{*}{ Psychotropic drugs } & \multirow{2}{*}{ Mean } & \multirow{2}{*}{$\mathrm{SD}$} & \multicolumn{2}{|c|}{$95 \%$ confidence interval } & \multirow{2}{*}{$\begin{array}{l}\text { Score } \\
9(\%)\end{array}$} & \multirow{2}{*}{ p-value } & \multirow{2}{*}{ Choice } \\
\hline & & & Lower & Upper & & & \\
\hline \multicolumn{8}{|l|}{ SSRIs } \\
\hline Escitalopram & 8.1 & 0.1 & 7.9 & 8.3 & 40.9 & 0.000 & First-line \\
\hline Paroxetine & 7.7 & 0.1 & 7.4 & 8.0 & 22.7 & 0.000 & First-line \\
\hline Sertraline & 7.5 & 0.2 & 7.2 & 7.9 & 23.0 & 0.000 & First-line \\
\hline Fluoxetine & 6.7 & 0.2 & 6.3 & 7.0 & 9.7 & 0.000 & Upper second-line \\
\hline Fluvoxamine & 6.0 & 0.3 & 5.5 & 6.5 & 5.4 & 0.000 & Upper second-line \\
\hline \multicolumn{8}{|l|}{ SNRIs } \\
\hline Venlafaxine & 7.6 & 0.1 & 7.3 & 7.9 & 25.4 & 0.000 & First-line \\
\hline Duloxetine & 7.0 & 0.2 & 6.6 & 7.4 & 8.9 & 0.000 & First-line \\
\hline Milnacipran & 5.9 & 0.2 & 5.4 & 6.3 & 0.0 & 0.000 & Lower second-line \\
\hline \multicolumn{8}{|l|}{ TCAs } \\
\hline Imipramine & 4.8 & 0.3 & 4.3 & 5.3 & 2.3 & 0.000 & Lower second-line \\
\hline Clomipramine & 4.7 & 0.3 & 4.1 & 5.3 & 2.7 & 0.000 & Lower second-line \\
\hline Nortriptyline & 4.7 & 0.3 & 4.1 & 5.3 & 2.9 & 0.000 & Lower second-line \\
\hline Amitriptyline & 4.6 & 0.3 & 4.0 & 5.2 & 2.3 & 0.000 & Lower second-line \\
\hline \multicolumn{8}{|l|}{ Other antidepressants } \\
\hline Mirtazapine & 5.8 & 0.2 & 5.3 & 6.2 & 0.0 & 0.000 & Lower second-line \\
\hline Moclobemide & 5.5 & 0.4 & 4.8 & 6.3 & 8.6 & 0.002 & Lower second-line \\
\hline Bupropion & 4.8 & 0.2 & 4.4 & 5.2 & 0.0 & 0.000 & Lower second-line \\
\hline Tianeptine & 4.7 & 0.3 & 4.0 & 5.3 & 0.0 & 0.000 & Lower second-line \\
\hline \multicolumn{8}{|l|}{ Benzodiazepines } \\
\hline Alprazolam & 6.7 & 0.2 & 6.3 & 7.1 & 10.6 & 0.000 & Upper second-line \\
\hline Clonazepam & 6.6 & 0.2 & 6.3 & 7.0 & 9.2 & 0.000 & Upper second-line \\
\hline Lorazepam & 5.9 & 0.2 & 5.5 & 6.2 & 3.2 & 0.000 & Lower second-line \\
\hline Diazepam & 5.5 & 0.2 & 5.1 & 5.9 & 1.6 & 0.000 & Lower second-line \\
\hline Bromazepam & 5.7 & 0.4 & 5.0 & 6.4 & 9.1 & 0.000 & Lower second-line \\
\hline \multicolumn{8}{|l|}{$\beta$-blockers } \\
\hline Propranolol & 7.1 & 0.2 & 6.6 & 7.5 & 19.7 & 0.000 & First-line \\
\hline \multicolumn{8}{|l|}{ Anticonvulsants } \\
\hline Valproic acid & 3.8 & 0.3 & 3.2 & 4.4 & 0.0 & 0.000 & Third-line \\
\hline Lamotrigine & 3.2 & 0.3 & 2.5 & 3.8 & 0.0 & 0.000 & Third-line \\
\hline Topiramate & 3.0 & 0.3 & 2.3 & 3.7 & 0.0 & 0.000 & Third-line \\
\hline \multicolumn{8}{|l|}{ Atypical antipsychotics } \\
\hline Quetiapine & 5.3 & 0.3 & 4.8 & 5.8 & 3.7 & 0.000 & Lower second-line \\
\hline Olanzapine & 4.7 & 0.3 & 4.2 & 5.3 & 0.0 & 0.000 & Lower second-line \\
\hline Aripiprazole & 4.8 & 0.3 & 4.2 & 5.5 & 5.0 & 0.000 & Lower second-line \\
\hline Risperidone & 4.3 & 0.3 & 3.7 & 4.8 & 4.4 & 0.000 & Lower second-line \\
\hline Amisulpride & 3.6 & 0.3 & 3.0 & 4.3 & 2.9 & 0.000 & Third-line \\
\hline \multicolumn{8}{|l|}{ Typical antipsychotics } \\
\hline Perphenazine & 3.4 & 0.4 & 2.7 & 4.1 & 2.9 & 0.000 & Third-line \\
\hline Chlorpromazine & 1.8 & 0.2 & 1.3 & 2.3 & 0.0 & 0.000 & Third-line \\
\hline \multicolumn{8}{|l|}{ Other } \\
\hline Buspirone & 5.8 & 0.2 & 5.4 & 6.2 & 6.5 & 0.000 & Lower second-line \\
\hline
\end{tabular}

SSRI: selective serotonin reuptake inhibitor, SNRI: serotonin-norepinephrine reuptake inhibitor, TCA: tricyclic antidepressant 
sant medication was agreed upon as an upper second-line choice. However, in individual drug surveys, venlafaxine and duloxetine were both agreed upon as first-line options. After reviewing the survey results, the authors' agreed to downgrade duloxetine to an upper second-line option, as it was only upgraded because it is also an SNRI.

Among the initial treatment regimens, standing or as-need- ed concomitant use of beta-blockers or benzodiazepines with antidepressants was agreed upon as the first-line option. However, only propranolol, a beta-blocker, was agreed upon as a first-line option in an individual drug survey. Among drugs in the benzodiazepine class, alprazolam and clonazepam were agreed upon as upper second- line options.

Among SSRI antidepressants, fluoxetine and fluvoxamine

Table 3. Guidelines on the initial treatment of social anxiety disorder

\begin{tabular}{|c|c|c|c|}
\hline \multirow{2}{*}{$\frac{\text { Recommendation grad }}{\text { Treatment regimens }}$} & \multicolumn{3}{|c|}{ Treatment } \\
\hline & & & \\
\hline First-line & \multicolumn{3}{|c|}{$\begin{array}{l}\text { SSRI only, SNRI(venlafaxine) only, Antidep+BDZ st, Antidep+BDZ as, Antidep+ } \beta \text {-blocker as, } \\
\text { Antidep+ } \beta \text {-blocker st, Antidep+BDZ st }+\beta \text {-blocker as, Antidep }+\mathrm{BDZ} \text { as }+\beta \text {-blocker as }\end{array}$} \\
\hline Upper second-line & \multicolumn{3}{|c|}{ SNRI(duloxetine) only, Antidep $+\mathrm{BDZ}$ st $+\beta$-blocker st, Antidep $+\mathrm{BDZ}$ as $+\beta$-blocker st } \\
\hline Lower second-line & \multicolumn{3}{|c|}{$\begin{array}{l}\text { SNRI(milnacipran) only, NaSSA only, Buspirone, } \beta \text {-blocker as only, BDZ as }+\beta \text {-blocker as, RIMA only, } \\
\text { BDZ st+ } \beta \text {-blocker st, BDZ as only, BDZ st }+\beta \text {-blocker as, Atypical antipsychotics, BDZ as }+\beta \text {-blocker st, } \\
\beta \text {-blocker st only, BDZ st only, TCA only }\end{array}$} \\
\hline Third-line & \multicolumn{3}{|c|}{ Anticonvulsants, Typical antipsychotics } \\
\hline \multicolumn{4}{|l|}{ Psychotropic drugs } \\
\hline First-line & \multicolumn{3}{|c|}{ Escitalopram, Paroxetine, Sertraline, Venlafaxine, Propranolol } \\
\hline Upper second-line & \multicolumn{3}{|c|}{ Fluoxetine, Fluvoxamine, Duloxetine, Alprazolam, Clonazepam } \\
\hline Lower second-line & \multicolumn{3}{|c|}{$\begin{array}{l}\text { Milnacipran, Imipramine, Clomipramine, Nortriptyline, Amitriptyline, Mirtazapine, Moclobemide, Bupropion, } \\
\text { Tianeptine, Lorazepam, Diazepam, Bromazepam, Quetiapine, Olanzapine, Aripiprazole, Risperidone, Buspirone }\end{array}$} \\
\hline Third-line & \multicolumn{3}{|c|}{ Valproic acid, Lamotrigine, Topiramate, Amisulpride, Perphenazine, Chlorpromazine } \\
\hline \multicolumn{4}{|c|}{$\begin{array}{l}\text { SSRI: selective serotonin reuptake inhibitor, Antidep: antidepressant, } \beta \text {-blocker as: } \beta \text {-blocker as-needed basis, BDZ: benzodiazepine, BDZ st: } \\
\text { BDZ standing basis, BDZ as: BDZ as-needed basis, } \beta \text {-blocker st: } \beta \text {-blocker standing basis, SNRI: serotonin-norepinephrine reuptake inhibi- } \\
\text { tor, NaSSA: noradrenergic and specific serotonergic antidepressant, RIMA: reversible inhibitor of monoamine oxidase A, TCA: tricyclic anti- } \\
\text { depressant }\end{array}$} \\
\hline \multirow{2}{*}{ Psychotropic drugs } & Initial starting dose $(\mathrm{mg})$ & Maintenance dose (mg) & Maximum possible dose (mg) \\
\hline & Mean \pm SD & Mean \pm SD & Mean \pm SD \\
\hline Escitalopram & $7 \pm 3.8$ & $15.7 \pm 5.7$ & $26.4 \pm 12.2$ \\
\hline Fluoxetine & $13.8 \pm 4.9$ & $29.7 \pm 11.2$ & $55.5 \pm 17.6$ \\
\hline Paroxetine IR & $12 \pm 4.6$ & $27.7 \pm 10.0$ & $46 \pm 13.1$ \\
\hline Paroxetine CR & $12.7 \pm 2.1$ & $31.3 \pm 11.1$ & $48.8 \pm 13.5$ \\
\hline Sertraline & $38 \pm 13.0$ & $107.7 \pm 51.9$ & $175.9 \pm 64.5$ \\
\hline Venlafaxine XR & $41.3 \pm 12.8$ & $146 \pm 48.9$ & $212.2 \pm 54.6$ \\
\hline Duloxetine & $30.3 \pm 1.6$ & $64.2 \pm 20.6$ & $94.9 \pm 27.9$ \\
\hline Mirtazapine & $9.7 \pm 3.7$ & $24.3 \pm 9.2$ & $43.9 \pm 11.5$ \\
\hline Alprazolam & $0.3 \pm 0.2$ & $0.8 \pm 0.4$ & $1.9 \pm 1.2$ \\
\hline Clonazepam & $0.4 \pm 0.2$ & $1 \pm 0.5$ & $2 \pm 1.1$ \\
\hline Diazepam & $3.1 \pm 1.4$ & $8.3 \pm 3.8$ & $16.6 \pm 9.0$ \\
\hline Lorazepam & $0.7 \pm 0.4$ & $1.5 \pm 0.7$ & $3.1 \pm 2.3$ \\
\hline Propranolol & $19.3 \pm 8.8$ & $44.7 \pm 22.7$ & $84.9 \pm 36.7$ \\
\hline Olanzapine & $3.3 \pm 1.3$ & $6.4 \pm 3.1$ & $13.7 \pm 6.3$ \\
\hline Quetiapine & $23.2 \pm 14.2$ & $100.9 \pm 116.5$ & $309.6 \pm 269.8$ \\
\hline Buspirone & $10.8 \pm 4.4$ & $23.5 \pm 9.6$ & $39.5 \pm 17.7$ \\
\hline
\end{tabular}

Paroxetine IR: paroxetine immediate release, Paroxetine CR: paroxetine controlled release, venlafaxine XR: venlafaxine extended release 
were agreed upon as upper second-line options.

Table 3 includes final guidelines for treatment regimens and specific drugs.

\section{Psychotropic drug dose in the initial stage of treatment}

Table 4 includes results regarding the commencing dose, maintenance dose, and maximum dose of medication during the initial stages of SAD treatment.

\section{Duration of psychotropic drug use during initial treatment}

Table 5 includes results regarding the evaluation period, the duration of maintenance treatment, the duration of the tapering phase following maintenance treatment, and the duration of treatment upon recurrence of SAD symptoms after pharmacological intervention.

\section{Review including public hearing and guideline compilation}

The hearing was held on November 15, 2013, at the Fall Academic Meeting of the Korean Academy of Anxiety Disorder. The final guidelines were distributed on October 30, 2015, at the Fall Academic Meeting of the Korean Academy of Anxiety Disorder, following which a symposium and briefing session were held.

\section{DISCUSSION}

The purpose of the present study was to present a clinical basis for the treatment of SAD. We investigated initial treatment strategies for SAD in order to develop medication guidelines. Recommended first-line regimens included either monotherapy with SSRIs or an SNRI (venlafaxine), or concomitant use of antidepressants with beta-blockers or benzodiazepines. First-line medication choices included escitalopram, paroxetine, sertraline, venlafaxine, and propranolol.

The WFSBP guideline, upon which we relied heavily in preparing the questionnaire utilized in the present study, recommends the SSRIs (e.g., escitalopram, fluvoxamine, paroxetine, sertraline) and the SNRI (e.g., venlafaxine) as grade 1 treatments. The monoamine oxidase inhibitor (MAOI, phenel- zine) is recommended as a grade 2 treatment, while citalopram, benzodiazepines (e.g., clonazepam), and gabapentin are recommended as grade 3 treatments. ${ }^{8}$ In addition to SSRIs and SNRI such as venlafaxine, the British guideline ${ }^{13}$-published slightly before the WFSBP guideline-reviewed the potential for a wide variety of psychotropic drugs in the pharmacological treatment of SAD, including phenelzine, moclobemide, benzodiazepines (e.g., bromazepam, clonazepam), anticonvulsants (e.g., gabapentin, pregabalin), olanzapine, imipramine, buspirone, and atenolol, among others. However, the Canadian guideline published by Swinson et al. ${ }^{9}$ and the Australian guideline, ${ }^{11}$ which were published around the same time as the WFSBP guideline, do not recommend the use of imipramine, beta-blockers (propranolol, atenolol), or buspirone.

The German guideline, ${ }^{16}$ published during the preparation of our guidelines, relied on the methods for classifying evidence reported by Eccles and Mason. ${ }^{17}$ The guideline recommends the use of escitalopram, paroxetine, sertraline, venlafaxine (grade A), or moclobemide (clinical agreement) as first-line psychotropic medications, though benzodiazepines are not recommended due to side effects such as dependence, among others. Also the Canadian guideline ${ }^{18}$ published around the same time, regards escitalopram, fluvoxamine, fluvoxamine controlled release (CR), paroxetine, paroxetine controlled release (CR), pregabalin, sertraline, and venlafaxine extended release (XR) as first-line treatment options; gabapentin, phenelzine, and benzodiazepines as second-line treatment options; and duloxetine, fluoxetine, mirtazapine, and moclobemide as third-line treatment options. Beta-blockers such as atenolol and propranolol are not recommended.

In the preparation of this guideline, wherein the consensus of clinicians was important, it was necessary to consider that there existed characteristics of pharmacological treatment specific to the situation of each country, and that this guideline reflected the domestic clinical reality as of 2013. In most cases, anxiolytics such as benzodiazepines were not recommended in foreign guidelines; however, in the present study, respondents agreed that such agents could be used concomitantly with antidepressants. This difference likely arose from different standards of practice among countries: Most foreign guidelines recommend monotherapy; however, in this study, the treatment strategies including combination therapy were

Table 5. Assessment, maintenance, and termination duration of pharmacological treatment for social anxiety disorder

\begin{tabular}{lcc}
\hline \multicolumn{1}{c}{ Division } & \multicolumn{2}{c}{ Duration } \\
\cline { 2 - 3 } & Mean \pm SD & Median (Q1-Q3) \\
\hline Evaluation period for initial treatment efficacy (weeks) & $4.8 \pm 2.4$ & $4(4-6)$ \\
Duration of the maintenance therapy (months) & $11.2 \pm 4.7$ & $12(6-12)$ \\
Medication tapering phase after the maintenance therapy (weeks) & $15.6 \pm 7.1$ & $12(12-24)$ \\
Treatment duration for recurrence (months) & $21.6 \pm 8.5$ & $24(12-24)$ \\
\hline
\end{tabular}


inquired to reflect the domestic reality, and the results of this study reflected the expectations for faster treatment effect in Korea. In the regimen survey, benzodiazepines were agreed upon as a first-line option only when used concomitantly with an antidepressant. Meanwhile, in the psychotropic drug survey, benzodiazepines were not agreed upon as a first-line option, indicating that domestic clinicians were well aware of the side effects of benzodiazepines, such as tolerance and dependence. Therefore, the following phrase was included in the final version of the guideline: "Benzodiazepines can be used for early symptom relief during the initial stages of treatment with first-line antidepressants-at which point the full effect of antidepressants may not be apparent. However, this medication should be utilized for a short period of time, and symptoms should be monitored closely, paying particular attention to possible side effects."

Unlike the above-mentioned foreign reference guidelines, the study results of such agreement to use the beta-blocker even though it was used only as a combination therapy with antidepressants, suggested the tendency of domestic clinicians to prefer the use of beta-blockers for contol of symptoms during earlier stagies of treatment, before the effects of antidepressant medications become apparent. This result is in contrast with the foreign guideline, ${ }^{8}$ which permits the use of betablockers for performance anxiety, though they are not recommended for SAD. Thus, these findings again reflect the domestic clinical reality of perceiving performance anxiety as a continuation of symptoms, suggesting the instability of SAD diagnosis. Among all beta-blockers, only propranolol was preferred by clinicians because it has fewer side effects than other beta-blockers such as atenolol. However, as propranolol may cause hypotension, the following statement was included in the guidelines: "Long-term study results associated with betablockers are not sufficient, and this medication should be used with caution due to the potential side effects."

In addition, our findings revealed that fluvoxamine, phenelzine, and moclobemide-relatively widely used in foreign countries-were not preferred by clinicians in Korea. Due to a lack of evidence regarding the use of antiepileptics for the treatment of SAD in Korea, we did not consider such treatment as a subject of consensus.

Atypical antipsychotics including quetiapine were agreed upon as lower second-line treatment options, in accordance with the Australian guideline, ${ }^{11}$ which regards quetiapine and olanzapine as second-line treatment options. Furthermore, our findings were similar to those of Stein et al., ${ }^{12}$ which do not regard quetiapine as a first-line treatment because it may aggravate social anxiety and result in side effects.

In the present study, we observed similar pharmacological treatment doses among Korean and foreign clinicians. How- ever, especially with SSRIs and SNRIs, Korean clinicians tended to initiate treatment at a lower dose and steadily titrate to a therapeutic dose, while monitoring for potential side effects, relative to clinicians abroad.

Further, in the present study, domestic clinicians considered the duration for assessing the efficacy of the initial treatment to be approximately 5 weeks, in contrast to the duration of 12 weeks recommended by Stein et al. ${ }^{12}$ However, our findings aligned with the most recently proposed recommendation of Bandelow et al. ${ }^{16}$ In Korea, such results showed that clinicians determined the effects of the drug at an earlier stage.

While respondents of the present study reported that approximately 11 months of maintenance therapy was recommended, British guidelines ${ }^{13}$ recommend a maintenance period of 6 months or more, as increases in efficacy and decreases in recurrence are observed up to 6 months following the initiation of treatment. In accordance with these findings, a metaanalysis by Katzman et al. ${ }^{18}$ revealed maintenance periods of 6 months or more were associated with significant improvements in the prevention of recurrence. However, Swinson et al. ${ }^{9}$ reported that pharmacological treatment should be continued for 12-24 months, while Bandelow et al.$^{16}$ concluded that medication should be continued for 6-12 months or longer after remission begins. The results of the present study indicated that domestic clinicians preferred a similar duration of maintenance treatment for SAD when compared with foreign clinicians.

Although this study presented the first guidelines regarding the treatment of SAD in Korea, the following limitations should be noted. First, although we aimed to follow the evidence-based algorithm method for developing guidelines previously outlined by Woolf, ${ }^{19}$ clinical evidence regarding the pharmacological treatment of SAD in Korea is rather scarce; therefore, we mainly utilized the expert consensus algorithm method for the development of our guidelines. In future, further studies of SAD are required to develop more complete practice guidelines. Second, psychotherapy methods such as cognitive behavioral therapy (CBT), which may be effective in the treatment of SAD, were not included. Many foreign guidelines discuss methods such as CBT for the treatment of SAD. However, in Korea, there were no sufficient experts in this field at the time of this study, so our guidelines focused only on pharmaceutical therapy. Third, we developed these guidelines when the DSM-IV-TR was used as the diagnostic standard, just prior to the release of the DSM-5.

\section{Acknowledgments}

This work was supported by 2011 the Korean Academy of Anxiety Disorder grant. 


\section{REFERENCES}

1. American Psychiatric Association. Diagnostic and Statistical Manual of Mental Disorders. 4th ed. Washington, DC: American Psychiatric Press; 1994.

2. Seoul National University Hospital. The Epidemiological Survey of Psychiatric Illness in Korea 2011. Seoul: Ministry of Health and Welfare in Korea; 2011.

3. Hwu HG, Yey EK, Chang LY. Prevalence of psychiatric disorders in Taiwan defined by the Chinese Diagnostic Interview Schedule. Acta Psychiatr Scand 1989;79:136-147.

4. Furmark T. Social phobia: overview of community surveys. Acta Psychiatr Scand 2002;105:84-93.

5. Kessler RC, Wang PS. The descriptive epidemiology of commonly occurring mental disorders in the United States. Annu Rev Public Health 2008;29:115-129.

6. Beesdo K, Bittner A, Pine DS, Stein MB, Höfler M, Lieb R, et al. Incidence of social anxiety disorder and the consistent risk for secondary depression in the first three decades of life. Arch Gen Psychiatry 2007; 64:903-912.

7. Kim DJ, Ahn YM, Kang DY, Kim SH, Kim CY, Min KJ, et al. Korean Medication Algorithm Projects for major psychiatric disorders (II): Background, basic plan, organization of KMAP and principles, methods of algorithm development and limitation, caution when using KMAP. Korean J Psychopharmacol 2002;13:30-36.

8. Bandelow B, Zohar J, Hollander E, Kasper S, Möller HJ; WFSBP Task Force on Treatment Guidelines for Anxiety, Obsessive-Compulsive and Post-Traumatic Stress Disoders, et al. World Federation of Societies of Biological Psychiatry (WFSBP) guidelines for the pharmacological treatment of anxiety, obsessive-compulsive and post-traumatic stress disorders - first revision. World J Biol Psychiatry 2008;9:248-312.

9. Swinson RP, Antony MM, Bleau P, Chokka P, Craven M, Fallu A, et al. Clinical practice guidelines - management of anxiety disorders. Can J Psychiatry 2006;51 (8 Suppl 2):9S-91S.

10. Montgomery SA, Lecrubier Y, Baldwin DS, Kasper S, Lader M, Nil R, et al. ECNP Consensus Meeting, March 2003. Guidelines for the investigation of efficacy in social anxiety disorder. Eur Neuropsychopharmacol 2004;14:425-433.

11. Western Australian Psychotropic Drugs Committee. Anxiety Disorders Drug Treatment Guidelines. Sydney: Watag; 2008.

12. Stein DJ, Baldwin DS, Bandelow B, Blanco C, Fontenelle LF, Lee Sing, et al. A 2010 evidence-based algorithm for the pharmacotherapy of social anxiety disorder. Curr Psychiatry Rep 2010;12:471-477.

13. Baldwin DS, Anderson IM, Nutt DJ, Bandelow B, Bond A, Davidson JRT, et al. Evidence-based guidelines for the pharmacological treatment of anxiety disorders: recommendations from the British Association for Psychopharmacology. J Psychopharmacol 2005;19:567-596.

14. Bandelow B, Zohar J, Hollander E, Kasper S, Möller HJ; World Federation of Societies of Biological Psychiatry Task Force on Treatment Guidelines for Anxiety, et al. World Federation of Societies of Biological Psychiatry (WFSBP) guidelines for the pharmacological treatment of anxiety, obsessive-compulsive and posttraumatic stress disorders. World J Biol Psychiatry 2002;3:171-199.

15. National Collaborating Centre for Mental Health. Social Anxiety Disorder: The NICE Guideline on Recognition, Assessment and Treatment. London: Royal College of Psychiatrists; 2013.

16. Bandelow B, Lichte T, Rudolf S, Wiltink J, Beutel ME. The diagnosis of and treatment recommendations for anxiety disorders. Dtsch Arztebl Int 2014;111:473-480.

17. Eccles M, Mason J. How to develop cost-conscious guidelines. Health Technol Assess 2001;5:1-69.

18. Katzman MA, Bleau P, Blier P, Chokka P, Kjernisted K, Van Ameringen M; Canadian Anxiety Guidelines Initiative Group on behalf of the Anxiety Disorders Association of Canada/Association Canadienne des troubles anxieux and McGill University. Canadian clinical practice guidelines for the management of anxiety, posttraumatic stress and obsessive-compulsive disorders. BMC Psychiatry 2014;14(Suppl 1):S1.

19. Woolf SH. Practice guidelines, a new reality in medicine: II. Methods of developing guidelines. Arch Intern Med 1992;152:946-952. 